\title{
Corrigendum: Molecular imaging of hydrogen peroxide produced for cell signaling
}

Evan W Miller, Orapim Tulyathan, Ehud Y Isacoff \& Christopher J Chang

Nature Chemical Biology 3, 263-267 (2007); published online 1 April 2007; corrected after print 3 May 2007

In the version of this article initially published, the second author's last name is misspelled. The author's name should read Orapim Tulyathan. The error has been corrected in the HTML and PDF versions of the article. 